\title{
Reward-Related Behavioral, Neurochemical and Electrophysiological Changes in a Rat Model of Autism Based on Prenatal Exposure to Valproic Acid
}

\author{
Sara Schiavi ${ }^{1 \dagger}$, Daniela lezzi ${ }^{2 \dagger}$, Antonia Manduca ${ }^{1}$, Stefano Leone ${ }^{1}$, Francesca Melancia ${ }^{1}$, \\ Carmen Carbone ${ }^{2}$, Michele Petrella ${ }^{3}$, Guido Mannaioni ${ }^{2}$, Alessio Masi ${ }^{2,3 * t}$ \\ and Viviana Trezza ${ }^{1 * t}$ \\ ${ }^{1}$ Department of Science, Section of Biomedical Sciences and Technologies, University "Roma Tre", Rome, Italy, ${ }^{2}$ Department \\ of Neuroscience, Psychology, Drug Research and Child Health -NEUROFARBA-, Section of Pharmacology and Toxicology, \\ School of Psychology, University of Florence, Florence, Italy, ${ }^{3}$ School of Pharmacy, University of Camerino, Camerino, Italy
}

OPEN ACCESS

Edited by:

João Peça,

University of Coimbra, Portugal

Reviewed by:

Rong Zhang,

Neuroscience Research Institute,

Peking University, China

Victorio Bambini-Junior,

University of Central Lancashire,

United Kingdom

*Correspondence:

Alessio Masi

alessio.masi@unifi.it

Viviana Trezza

viviana.trezza@uniroma3.it

${ }^{\dagger}$ These authors have contributed equally to this work

Received: 20 June 2019 Accepted: 10 October 2019 Published: 25 October 2019

Citation:

Schiavi S, lezzi D, Manduca A, Leone S, Melancia F, Carbone C,

Petrella M, Mannaioni G, Masi A and

Trezza V (2019) Reward-Related

Behavioral, Neurochemical and Electrophysiological Changes in a Rat Model of Autism Based on Prenatal

Exposure to Valproic Acid.

Front. Cell. Neurosci. 13:479.

doi: 10.3389/fncel.2019.00479
Prenatal exposure to the antiepileptic drug valproic acid (VPA) induces autism spectrum disorder (ASD) in humans and autistic-like behaviors in rodents, which makes it a good model to study the neural underpinnings of ASD. Rats prenatally exposed to VPA show profound deficits in the social domain. The altered social behavior displayed by VPA-exposed rats may be due to either a deficit in social reward processing or to a more general inability to properly understand and respond to social signals. To address this issue, we performed behavioral, electrophysiological and neurochemical experiments and tested the involvement of the brain reward system in the social dysfunctions displayed by rats prenatally exposed to VPA (500 mg/kg). We found that, compared to control animals, VPA-exposed rats showed reduced play responsiveness together with impaired sociability in the three-chamber test and altered social discrimination abilities. In addition, VPA-exposed rats showed altered expression of dopamine receptors together with inherent hyperexcitability of medium spiny neurons (MSNs) in the nucleus accumbens (NAc). However, when tested for socially-induced conditioned place preference, locomotor response to amphetamine and sucrose preference, control and VPA-exposed rats performed similarly, indicating normal responses to social, drug and food rewards. On the basis of the results obtained, we hypothesize that social dysfunctions displayed by VPA-exposed rats are more likely caused by alterations in cognitive aspects of the social interaction, such as the interpretation and reciprocation of social stimuli and/or the ability to adjust the social behavior of the individual to the changing circumstances in the social and physical environment, rather than to inability to enjoy the pleasurable aspects of the social interaction. The observed neurochemical and electrophysiological alterations in the NAc may contribute to the inability of VPA-exposed rats to process and respond to social cues, or, alternatively, represent a compensatory mechanism towards VPA-induced neurodevelopmental insults.

Keywords: autism, valproate, social play behavior, dopamine, electrophysiology 


\section{INTRODUCTION}

Although the precise causes of autism spectrum disorder (ASD) are still the subject of significant debate, a number of factors (rare gene mutations, gene variations and adverse environmental events) have been identified that, interacting in complex ways, affect early brain development contributing to the risk of ASD. Among the environmental factors involved in the pathogenesis of ASD, it has been well documented that prenatal exposure to the antiepileptic drug valproic acid (VPA) is associated with increased risk of neurodevelopmental delay and autistic symptoms in the offspring. Indeed, when given during gestation, VPA not only increases the risk for various congenital malformations (Kozma, 2001; Kini et al., 2006), but also induces core autistic symptoms in the offspring, i.e., impaired communication, reduced sociability and stereotyped behaviors (Williams and Hersh, 1997; Williams et al., 2001). Based on this clinical evidence, prenatal exposure to VPA in rodents has been validated as a drug-induced preclinical model of ASD (Roullet et al., 2013; Nicolini and Fahnestock, 2018; Tartaglione et al., 2019). In agreement with the clinical data, rodents exposed to VPA during pregnancy show marked behavioral impairments resembling the core and secondary signs of ASD (Rodier et al., 1996; Narita et al., 2002; Miyazaki et al., 2005; Schneider and Przewłocki, 2005; Servadio et al., 2016; Melancia et al., 2018). Since impaired social interaction is a key feature of ASD, a valid animal model is expected to exhibit deficits in this behavioral domain. Rodents are highly social species that engage in complex patterns of social behavior such as parental care and social play behavior (Panksepp et al., 1984; Ricceri et al., 2007). Rats prenatally exposed to VPA show a wide range of deficits in the social domain. At infancy, they show deficits in social communication and social discrimination, i.e., they are unable to properly communicate with their mother and siblings when removed from the nest and cannot discriminate between a neutral scent and their own nest odor (Schneider and Przewłocki, 2005; Dufour-Rainfray et al., 2010; Favre et al., 2013; Servadio et al., 2016; Bronzuoli et al., 2018; Cartocci et al., 2018; Melancia et al., 2018). At adolescence, VPA-exposed rats display atypical patterns of social play behavior, that is the most characteristic and rewarding social activity displayed by young mammals: indeed, compared to control animals, rats prenatally exposed to VPA respond to play solicitation mainly by partial rotation and evasion, rather than reciprocating the playful interaction (Servadio et al., 2016; Melancia et al., 2018). The social deficits displayed by VPA-exposed rats are long lasting, since they also persist at adulthood (Schneider and Przewłocki, 2005; Schneider et al., 2006, 2008; Markram et al., 2008; Kim et al., 2011, 2014; Servadio et al., 2016, 2018; Hirsch et al., 2018; Melancia et al., 2018; Fontes-Dutra et al., 2019) and are evocative of the social disturbances displayed by autistic patients over the course of development.

The pervasive social deficits found in autistic patients have been initially explained in terms of cognitive impairments and inability to infer others' mental states. More recently, they have been related to blunted social reward processing, i.e., inability to enjoy and prolong reciprocal social interactions, which has been hypothesized to be the consequence of abnormal activity of the brain reward circuit in social contexts (Chevallier et al., 2012; Pellissier et al., 2018). Along this line, the social dysfunctions displayed by VPA-exposed rats may be caused by either their inability to properly understand and respond to social signals by the social partner or by a failure of their reward system to assign a positive value to the social experience. The aim of the present study was to address this issue by performing behavioral, neurochemical and electrophysiological experiments in rats prenatally exposed to VPA. In particular, we determined whether the social deficits displayed by VPA-exposed rats are associated with changes in more specific reward-related behaviors, including social, drug and food rewards. Furthermore, given the important role of corticolimbic dopamine in (social) reward processes (Gunaydin et al., 2014; Vanderschuren et al., 2016), we measured the expression of D1 and D2 dopamine receptors in the prefrontal cortex (PFC), dorsal striatum (DS), nucleus accumbens (NAc) and hippocampus (HIPP) of VPA-exposed rats, since these brain areas play an important role in the modulation of social behavior. Last, since activation of dopaminergic terminals in the NAc of rats during bouts of interaction with novel conspecifics has been reported (Robinson et al., 2002; Gunaydin et al., 2014) and given the important role of NAc dopamine in rewarding forms of social interaction such as social play (Manduca et al., 2016), we addressed the role of the NAc in the social impairment displayed by VPA-exposed rats by performing electrophysiological experiments in this brain area.

\section{MATERIALS AND METHODS}

\section{Animals}

Female Wistar rats (Charles River, Italy), weighing $250 \pm 15 \mathrm{~g}$, were mated overnight. The morning when spermatozoa were found was designated as gestational day 1. Pregnant rats were singly housed in Macrolon cages [40 (length) $\times 26$ (width) $\times 20$ (height) $\mathrm{cm}$ ], under controlled conditions (temperature $20-21^{\circ} \mathrm{C}, 55 \%-65 \%$ relative humidity and $12 / 12 \mathrm{~h}$ light cycle with lights on at 07:00 h). Food and water were available ad libitum. On gestational day 12.5, females received a single intraperitoneal injection of either VPA or saline (SAL). Newborn litters found up to 17:00 $\mathrm{h}$ were considered to be born on that day [postnatal day (PND) 0]. On PND 1, the litters were culled to eight animals (six males and two females), to reduce the litter size-induced variability in the growth and development of pups during the postnatal period. On PND 21, the pups were weaned and housed in groups of three. The experiments were carried out on the male offspring during adolescence (PNDs 35-40) and adulthood (PNDs 90-95). One male pup per litter from different litters per treatment group was used in each experiment. For the flow cytometric experiments, we used brain samples from the VPA- and SAL-exposed rats tested in the social play behavior, the three-chamber and the social discrimination tests. Other cohorts of VPA- and SAL-exposed rats were used for the electrophysiology experiments and to investigate amphetamine- 
induced hyperlocomotion, sucrose preference and sociallyinduced Conditioned Place Preference (sCPP). The exact sample size (n) for each experimental group/condition is indicated in the figure legends. The sample size was based on our previous experiments and power analysis performed with the software $G$ power.

The experiments were approved by the Italian Ministry of Health (Rome, Italy) and performed in agreement with the Animals in Research: Reporting in vivo Experiments (ARRIVE) guidelines (Kilkenny et al., 2010), with the guidelines released by the Italian Ministry of Health (D.L. 26/14) and the European Community Directive 2010/63/EU. In particular, the experimental protocol was approved by the Animal Care Committees of both Roma Tre and Florence Universities and by the Italian Ministry of Health (authorization numbers: 31-2019PR and 955/2015-PR).

\section{Drugs}

VPA (Cayman Chemical, Ann Arbor, MI, USA) was dissolved in saline at a concentration of $250 \mathrm{mg} / \mathrm{ml}$ and administered at a dose $(500 \mathrm{mg} / \mathrm{kg}$ ) and time (gestational day 12.5) that have been shown to induce autistic-like behavioral changes in the offspring (Servadio et al., 2016; Melancia et al., 2018). Amphetamine (AMPH, Research Biochemicals International) was dissolved in saline and administrated at the dose of $0.5 \mathrm{mg} / \mathrm{kg} 30 \mathrm{~min}$ before the open field test to both VPA- and SAL-exposed offspring. We used a dose of AMPH that is known to affect locomotor activity without inducing stereotyped behaviors (Fowler et al., 2003; Manduca et al., 2014).

\section{Behavioral Tests}

\section{Social Play Test}

The test was performed in a sound-attenuated chamber under dim light conditions, as previously described (Trezza and Vanderschuren, 2008, 2009). At PNDs 35-40, rats were individually habituated to the test cage for $10 \mathrm{~min}$ on the 2 days before testing. On the test day, the animals were isolated for $3 \mathrm{~h}$ before testing. The test consisted of placing each experimental rat together with an untreated animal for $15 \mathrm{~min}$ in the testing chamber. In rats, a bout of social play behavior starts with one rat soliciting ("pouncing") another animal, by attempting to nose or rub the nape of its neck. The animal that is pounced upon can respond in different ways: if the animal fully rotates to its dorsal surface, "pinning" is the result (one animal lying with its dorsal surface on the floor with the other animal standing over it), which is considered the most characteristic posture of social play behavior in rats. The following parameters were scored for each animal of a pair using the Observer 3.0 software (Noldus, The Netherlands): (1) number of pinning events; (2) number of pouncing events; (3) evasion (the animal that is pounced upon does not prolong the playful interaction but rather runs away); and (4) play responsiveness [the percentage of response to play solicitation, as the probability of an animal of being pinned in response to pouncing by the stimulus partner (Servadio et al., 2016)]. Time spent in social exploration (the total amount of time spent in non-playful forms of social interaction, like sniffing any part of the body of the test partner, including the anogenital area, or grooming any part of the partner body).

\section{Three-Chambers Test}

The test was performed as previously described (Servadio et al., 2016). The apparatus was a rectangular three-chamber box, with two lateral chambers $(30 \times 35 \times 35 \mathrm{~cm} ; 1 \times \mathrm{w} \times \mathrm{h})$ connected to a central chamber $(15 \times 35 \times 35 \mathrm{~cm} ; 1 \times \mathrm{w} \times \mathrm{h})$. Each lateral chamber contained a small Plexiglas cylindrical cage. At PND 90, each experimental rat was individually allowed to explore the three-chamber apparatus for $10 \mathrm{~min}$ and then confined in the central compartment. An unfamiliar stimulus animal was confined in a cage located in one chamber of the apparatus, while the cage in the other chamber was left empty. Both doors to the side chambers were then opened, allowing the experimental animal to explore the apparatus for $10 \mathrm{~min}$. The discrimination index was scored using the Observer 3.0 software (Noldus, The Netherlands) and was calculated as the difference in time spent sniffing the stimulus animal and the time spent exploring the empty chamber, expressed as the percentage ratio of the total time spent exploring both the stimulus animal and the empty chamber.

\section{Social Discrimination Test}

The test was performed as previously described (Melancia et al., 2018). Briefly, animals were isolated for 7 days before testing. The test consisted of a learning trial and a retrieval trial, which were separated by a $30 \mathrm{~min}$ intertrial interval. During the learning trial, a juvenile (30 days old) unfamiliar rat was introduced into the home cage of the experimental rat for $5 \mathrm{~min}$. The time spent by the experimental rat investigating (sniffing, allogrooming and following) the juvenile was measured. Thirty-minutes after, the juvenile used in the learning trial was returned to the same adult's cage together with a novel juvenile. The time spent by the adult exploring the novel and the familiar juveniles was monitored for $5 \mathrm{~min}$. The discrimination index was calculated as the difference in time exploring the novel and the familiar animal, expressed as the percentage ratio of the total time spent exploring both animals (Campolongo et al., 2007).

\section{Open Field Test}

To assess whether adolescent and adult VPA- and SAL-exposed rats similarly responded to AMPH-induced hyperlocomotion, animals from both experimental groups were tested for horizontal locomotor activity in a squared box [40 (length) $\times 40$ (width) $\times 60$ (height) $\mathrm{cm}$ ]. Each animal was placed in the central zone of the apparatus and allowed to explore for $30 \mathrm{~min}$. Total locomotor activity (expressed as the frequency of crossings in the arena) was analyzed during the 30-min test session. After each session, the apparatus was cleaned with ethanol $70 \%$.

\section{Sucrose Preference Test}

At both adolescence and adulthood, rats were tested for preference of a $2 \%$ sucrose solution, using a two-bottle choice procedure (Monteggia et al., 2007) with slight modifications. Subjects were housed singly for the 3 days of test. Rats were given two bottles, one of sucrose $(2 \%)$ and one of tap water. 
Every $24 \mathrm{~h}$ the amount of sucrose and water consumed was evaluated. To prevent potential location preference of drinking, the position of the bottles was changed every $24 \mathrm{~h}$. The preference for the sucrose solution was calculated as the percentage of sucrose solution ingested relative to the total amount of liquid consumed.

\section{Socially-Induced Conditioned Place Preference}

The sCPP test was performed at adolescence and adulthood as previously described (Wei et al., 2015). Briefly, rats were placed in an acrylic box [75 (length) $\times 35$ (width) $\times 35$ (height) $\mathrm{cm}$ ], divided into two chambers by a clear acrylic wall with a small opening. Each chamber contained different types of autoclaved, novel bedding (Sanyx Bio Ultra litter and Padovan Sandy Litter), which differed in texture and shade (white vs. darkbrown). A 30-min preconditioning test was used to establish any baseline preference for either of the two types of novel bedding. Individual rats with strong preference for either type of bedding were excluded (typically, those that spent more than $1.5 \times$ time on one bedding over the other). The next day, each experimental animal was assigned to a social cage with cage-mates to be conditioned to one type of novel bedding for $24 \mathrm{~h}(\mathrm{CS}+)$. Then, the experimental rat was moved to an isolated cage with the other type of bedding for $24 \mathrm{~h}$ (CS-). Bedding assignments were counterbalanced for an unbiased design. Animals were then tested alone for $30 \mathrm{~min}$ in the two-chambered box and the time spent in each chamber was calculated to determine post-conditioning preference for either type of bedding. Fresh bedding was used at each step and chambers were thoroughly cleaned between sessions with ethanol $70 \%$ to avoid olfactory confounders.

\section{Brain Samples Collection}

Rats were rapidly decapitated, and their brains were removed and cut into coronal slices on a cold plate. The PFC, DS, NAc and hippocampus (HIPP) were dissected by hand under microscopic control within 2 min as previously described (Hill et al., 2010; Trezza et al., 2012; Gray et al., 2015).

\section{Flow Cytometric Experiments}

Brain samples for each brain area were transferred with PBS into $1.5 \mathrm{ml}$ tube. After centrifugation, the pellet was digested with Trypsin $(0.1 \%)$ for $30 \mathrm{~min}$ at $37^{\circ} \mathrm{C}$ on slight agitation. Cell suspensions were filtered with CellTrics $(100 \mu \mathrm{m})$ and washed with $5 \mathrm{ml}$ of PBS. A little sample of suspension was analyzed for forward and side scatter parameter for neuron population quality control (Cruz et al., 2013). Each sample was fixed with PFA (1\%), permeabilized with ice-cold ethanol (70\%) and incubated for at least $2 \mathrm{~h}$ at $-20^{\circ} \mathrm{C}$. Next, cells were centrifuged, rehydrated with $1 \mathrm{ml}$ PBS/BSA (1\%)/Triton $(0.1 \%)$, aliquoted in 96 well tissue culture plates with conical bottom. Samples were incubated for $1 \mathrm{~h}$ RT with primary antibody anti-D1 (1:100 diluted, Novusbio AB81296) and D2 dopamine receptors (1:100 diluted, Santa Cruz SC-7523). The cells were washed and incubated with secondary antibody anti-rabbit and anti-goat Alexa 488 conjugated. All samples were counterstained with propidium iodide/RNase buffer for nuclei identification (with G0 cell cycle phase DNA content) and for singlet/doublets discrimination. Mean fluorescence intensity of 20,000 useful cellular events for each sample, was calculated.

\section{Whole-Cell Patch Clamp Recordings in Acute Brain Slices}

Preparation of acute brain slices was performed with established procedures (Carbone et al., 2017). In brief, 1-month old male Wistar rats were anesthetized with isoflurane and decapitated. The brain was quickly removed and glued to the bottom of a vibroslicer slicing chamber (Leica VT 1000S, Leica Microsystem, Wetzlar, Germany). Coronal brain slices $(250 \mu \mathrm{m})$ containing the NAc were cut in a slicing solution composed of (in $\mathrm{mM}$ ): Nmethyl-D-glucamine (92), 4-(2-hydroxyethyl)-1-piperazine-1ethanesulfonic acid (20), glucose (25), $\mathrm{NaHCO}_{3}(30), \mathrm{NaH}_{2} \mathrm{PO}_{4}$ (1.25), $\mathrm{KCl}$ (2.5), $\mathrm{MgSO}_{4}$ (10), $\mathrm{CaCl}_{2}$ (0.5). During slicing, the solution was kept cold and infused with a 95\% $\mathrm{O}_{2}+$ $5 \% \mathrm{CO}_{2}$ gas mixture. Slices were transferred to a warm $\left(34-35^{\circ} \mathrm{C}\right)$, carbo-oxygenated recovery bath containing artificial Cerebral Spinal Fluid (aCSF) of the following composition (in $m M): \mathrm{NaCl}$ (130), $\mathrm{KCl}$ (3.5), $\mathrm{NaH}_{2} \mathrm{PO}_{4}$ (1.25), $\mathrm{NaHCO}_{3}$ (25), glucose (10), $\mathrm{CaCl}_{2}$ (2) and $\mathrm{MgSO}_{4}$ (1). Slices were allowed to recover for at least $30 \mathrm{~min}$ prior to experiments. During recordings, a single slice was kept in a flow chamber positioned under the microscope objective and continuously perfused with warm $\left(34-35^{\circ} \mathrm{C}\right)$, carbo-oxygenated aCSF. Whole-cell pipettes were pulled from thin-walled borosilicate capillaries (Harvard Apparatus, London, UK) with a vertical puller (Narishige PP830, Narishige International Limited, London, UK) and back-filled with an intracellular solution containing (in $\mathrm{mM}$ ): $\mathrm{K}+$ methanesulfonate (120), $\mathrm{KCl}$ (15), HEPES (10), EGTA (0.1), $\mathrm{MgCl}_{2}$ (2), $\mathrm{Na}_{2}$ Phosphocreatine (5), $\mathrm{Na}_{2} \mathrm{GTP}$ (0.3) and MgATP (2), resulting in a bath resistance of 3-5 $\mathrm{M} \Omega$. Unless otherwise specified, all drugs were purchased from Sigma-Aldrich (St. Louis, MO, USA) and bath-applied. Access resistance was monitored during voltage clamp recordings with 100-ms, -10$\mathrm{mV}$ steps, throughout the experiment. Recordings undergoing a drift in access resistance $\geq 10 \%$ were discarded. No whole-cell compensations were used. Signals were sampled at $10 \mathrm{kHz}$ and low-pass filtered at $3 \mathrm{kHz}$ with an Axon Multiclamp 700B (Molecular Devices, Sunnyvale, CA, USA). NAc-MSNs were identified by their morphological and electrophysiological properties (Cepeda et al., 2008). To examine the effects of VPA prenatal exposure on medium spiny neuron (MSN) excitability, current clamp input-output curves were obtained by injecting 800 ms-current steps with amplitude ranging from -50 to $+550 \mathrm{pA}$ with $50 \mathrm{pA}$ increments. Inwardly rectifying potassium currents (IKir) were obtained by imposing 500 ms-voltage steps with amplitude ranging from -150 to $-60 \mathrm{mV}$ with $10 \mathrm{mV}$ increments. Potassium currents were recorded in the presence of tetrodotoxin (TTX, $1 \mu \mathrm{M}$, Tocris, Biosciences, Bristol, UK), a blocker of voltage-dependent sodium channels. IKir was isolated as the $1 \mathrm{mM} \mathrm{CsCl}$-sensitive component. IKir current density was obtained by normalizing current amplitudes for membrane capacitance (derived from the area underlying current peaks elicited by $-10 \mathrm{mV}$ steps) and expressed as $\mathrm{pA} / \mathrm{pF}$. 


\section{Statistical Analysis}

Behavioral and neurochemical data are expressed as mean \pm SEM. To assess the effects of the prenatal treatment (VPA or SAL) on the parameters measured, data were analyzed with Student's $t$-tests. Two-way analysis of variance (ANOVA) was used to assess the effects of prenatal and postnatal treatments in the open field test, using prenatal (VPA or SAL) and postnatal (AMPH or vehicle) treatments as between-subjects factor. Two-way ANOVA was followed by Student's-Newman-Keuls post hoc test where appropriate. All behavioral tests were scored by a trained observer who was unaware of treatment condition to reduce performance bias. Random allocation of animals to treatment groups and to behavioral tasks and blinding of investigators assessing outcomes were adopted to reduce selection and detection bias. All behavioral data were tested for normality.

For electrophysiological experiments, data are presented as mean \pm SEM of $n$ cells obtained from $\mathrm{N}$ animals. Statistical significance was assessed with student's $t$-test for unpaired samples (Microcal Origin 9.1; Northampton, MA, USA). Significance at the $P<0.05,0.01$ and 0.001 level is indicated with ${ }^{*},{ }^{* *}$ and ${ }^{* * *}$, respectively, in figures. Graphs and representative traces were generated with Microcal Origin 9.1. Example traces represent typical observations.

\section{RESULTS}

\section{Social Play Test}

VPA-exposed rats showed reduced play responsiveness compared with SAL-exposed animals $(t=3.67, p=0.002$, $d f=16$; Figure 1A). Indeed, while no differences were found between SAL- and VPA-exposed animals in the number of pinning $(t=0.81, p=$ n.s., $d f=16$; data not shown $)$ and pouncing $(t=-0.86, p=$ n.s., $d f=16$; data not shown $)$, VPA-exposed rats displayed a higher frequency of partial rotation $(t=-2.81$, $p=0.013, d f=16$; Figure 1B) compared to SAL-exposed animals. No differences in the total time spent in general social exploration were found between SAL- and VPA-exposed animals $(t=1.43, p=$ n.s.,$d f=16$, data not shown $)$.

\section{Three-Chambers Test}

VPA-exposed rats showed decreased sociability in the threechamber test, as they spent less time sniffing the stimulus animal compared to SAL-exposed rats, showing a lower discrimination index $(t=2.27, p=0.039, d f=14$; Figure 1C).

\section{Social Discrimination Test}

VPA-exposed animals showed impaired social discriminative abilities as they showed a lower discrimination index in the social discrimination test compared with SAL-exposed animals $(t=2.27, p=0.044, d f=14$; Figure 1D $)$.

\section{Amphetamine-Induced Hyperlocomotion}

AMPH increased locomotor activity of SAL- and VPA-exposed rats both at adolescence and adulthood. A two-way ANOVA analysis performed on the frequency of crossing after treatment with AMPH or its vehicle gave the following results: PNDs
35-40 $\left(F_{\text {(prenatal treat. }) 1,28}=1.56, p=\right.$ n.s.; $F_{\text {(treat.) } 1,28}=10.97$,

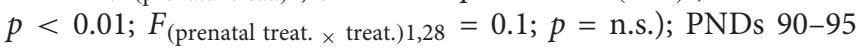
$\left(F_{\text {(prenatal treat.) }), 27}=1.40, p=\right.$ n.s.; $F_{\text {(treat.) }), 27}=33.05, p<0.001$; $F_{\text {(prenatal treat. } \times \text { treat.) } 1,27}=0.76 ; p=$ n.s.). Post hoc analysis revealed that $\mathrm{AMPH}$ increased the frequency of crossing both in SAL- (PNDs 35-40: $p<0.05$; PNDs 90-95: $p<0.001$ ) and VPA-exposed rats (PNDs 35-40: $p<0.05$; PNDs 90-95: $p=0.002$; Figures $2 \mathrm{~A}-\mathrm{D}$ ).

\section{Sucrose Preference Test}

Both SAL- and VPA-exposed rats preferred the sucrose over the water solution the in the sucrose preference test, at both PNDs 35-40 and 90-95 (PNDs 35-40: SAL: $t=-10.53, p<0.001$, $\mathrm{df}=16$; VPA: $t=-17.65, p<0.001, d f=12$, Figure 2B; PNDs 90-95: SAL: $t=-10.43, p<0.001, d f=18$; VPA: $t=-11.76$, $p<0.001, d f=18$; Figure 2E).

\section{Socially-Induced Conditioned Place Preference}

Both at adolescence and adulthood, VPA-exposed rats did not show deficits in the sCPP test. Indeed, during the test session animals of both experimental groups spent more time in the chamber containing the bedding used for the social conditioning (CS+; PNDs 35-40: Saline group: $t=-2.63, p<0.05, d f=22$; VPA group: $t=-3.31, p<0.01, d f=22$; Figure 2C; PNDs 90-95: Saline group: $t=-2.19, p<0.05, d f=14$; VPA group: $t=-2.55$, $p<0.05, d f=20$; Figure 2F).

\section{Flow Cytometric Analysis of Dopamine Receptors}

At adolescence, VPA-exposed rats shows a significative increase in D2 dopamine receptor expression in the NAc (SAL-exposed animals: MFI $=2,891$; VPA-exposed animals: $\mathrm{MFI}=5,272$, $p<0.05$; Figures 3A,B).

At adulthood, VPA-exposed rats showed a significative increase in D1 dopamine receptor expression in the NAc (SAL-exposed animals: MFI $=4,789$; VPA-exposed animals: MFI $=6,690, p=0.025$; Figure 3C) and HIPP (SAL-exposed animals: $\mathrm{MFI}=3,987$; VPA group: $\mathrm{MFI}=5,299, p=0.008$; Figure 3C), whereas D2 dopamine receptors were significative increased only in the NAc (SAL-exposed animals: $\mathrm{MFI}=1,697$; VPA-exposed animals: MFI $=2,152, p=0.022$; Figure 3D).

\section{Electrophysiological Recordings of NAC MSNs in Acute Brain slices}

Whole cell patch clamp recordings were obtained from NAc MSNs in acute coronal slices prepared from SAL- and VPA-exposed rats at PNDs 30-35 ( $N=9$ and 7, respectively, Figures $4 \mathbf{A}, \mathbf{B})$. While we found no differences in passive membrane properties between MSNs from SAL-exposed rats (SAL MSNs) and VPA-exposed rats (VPA MSNs; input resistance: $\mathrm{SAL}$ MSNs $=111.4 \pm 8.81 \mathrm{M} \Omega, n=22$; VPA MSNs $=139.2 \pm 12.78 \mathrm{M} \Omega, n=23$; membrane capacitance: SAL MSNs $=45.73 \pm 3.07 \mathrm{pF}, n=23$; VPA MSNs $=47.36 \pm 4.49 \mathrm{pF}$ $n=26$; threshold: SAL MSNs $=-39.43 \pm 1.3 \mathrm{mV}, n=35$; VPA MSNs $=-39.96 \pm 0.93 \mathrm{mV}, n=33 ; p=$ n.s.; all; Figure 4C), the latter group showed a significant depolarization 


\section{Adolescence}
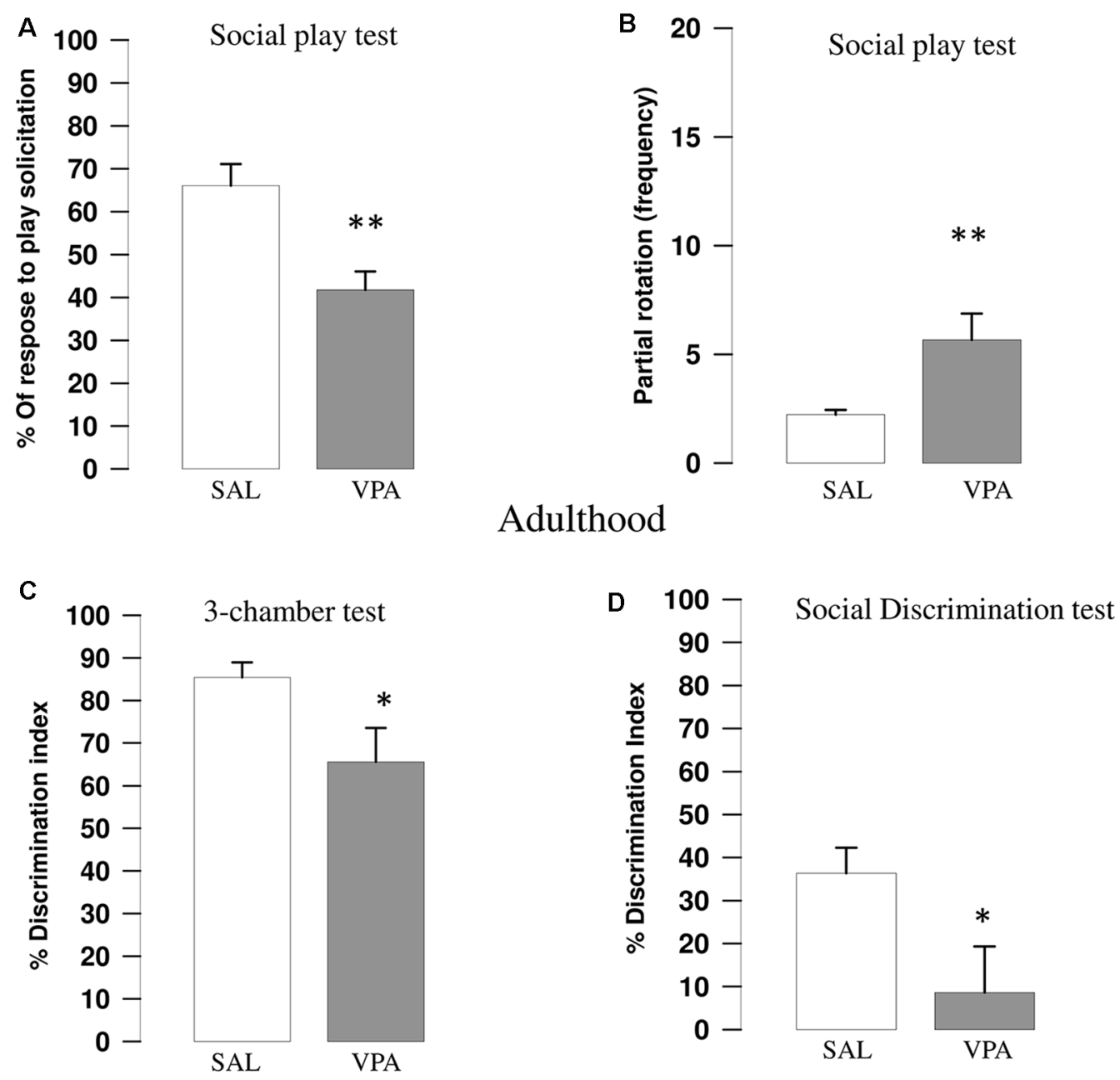

FIGURE 1 | Valproic acid (VPA)-exposed rats showed altered social play behavior, impaired sociability in the three-chamber test and reduced social discrimination abilities. VPA-exposed rats showed reduced response to play solicitation (A) and increased frequency of partial rotation (B; $n=\mathrm{SAL} 9, n=\mathrm{VPA} 9)$. VPA-exposed rats also showed reduced sociability in the three-chamber test $\mathbf{( C )}$, since they showed a lower discrimination index indicating (i.e., they spent less time sniffing the stimulus animal compared to SAL-exposed rats; $n=$ SAL $8, n=$ VPA 8). VPA-exposed rats also showed impaired social discrimination as they displayed a lower discrimination index compared to SAL-exposed animals in the social discrimination task (D; $n=\mathrm{SAL} 8, n=\mathrm{VPA} 8)$. Data represent mean values \pm SEM; ${ }^{*} p<0.05$, ** $p<0.01$, vs. SAL group (Student's $t$-test).

of the resting membrane potential as compared to SAL MSNs (SAL MSNs $=-77.59 \pm 0.83 \mathrm{mV}, n=35$; VPA MSNs $=-71.72 \pm 0.98 \mathrm{mV}, n=37 ; p=0.0008$; Figure 4C). We then determined the intrinsic excitability of MSNs in acute slices containing the NAc by measuring the number of action potentials elicited by depolarizing current steps of increasing amplitude. Figure $\mathbf{5 A}$ shows a direct comparison of representative voltage responses obtained at each value of imposed current from SAL and VPA MSNs. The minimal value of current amplitude required to elicit an action potential in VPA MSNs was significantly lower compared to SAL MSNs (100 pA vs. $200 \mathrm{pA}$ ). However, as the amplitude of the depolarizing current increased $(\geq 250 \mathrm{pA}$ ), while the number of APs fired by SAL MSNs increased linearly, VPA MSNs progressively lost the ability to fire action potentials. The plot in Figure 5B reports the mean number of APs \pm SEM fired by SAL (black) and VPA (green) MSNs vs. imposed current (SAL MSNs, $n=35$, vs. VPA MSNs $n=37 ; 150 \mathrm{pA}$; $p=0.01 ; 200 \mathrm{pA} ; p=0.03 ; 350 \mathrm{pA} ; p=0.02 ; \geq 400 \mathrm{pA} ;$ $p<0.0001)$. Depolarized resting potential and altered AP discharge pattern are consistent with a change in the density of whole-cell $\mathrm{K}^{+}$currents. Based on the reported relevance of the inward-rectifying $\mathrm{K}^{+}$current (IKir) in setting resting potential in MSNs (Kreitzer, 2009), we measured whole-cell currents elicited at hyperpolarizing potentials in control aCSF and in the presence of $1 \mathrm{mM} \mathrm{Cs}^{+}$, a IKir blocker (Cazorla et al., 2012). 


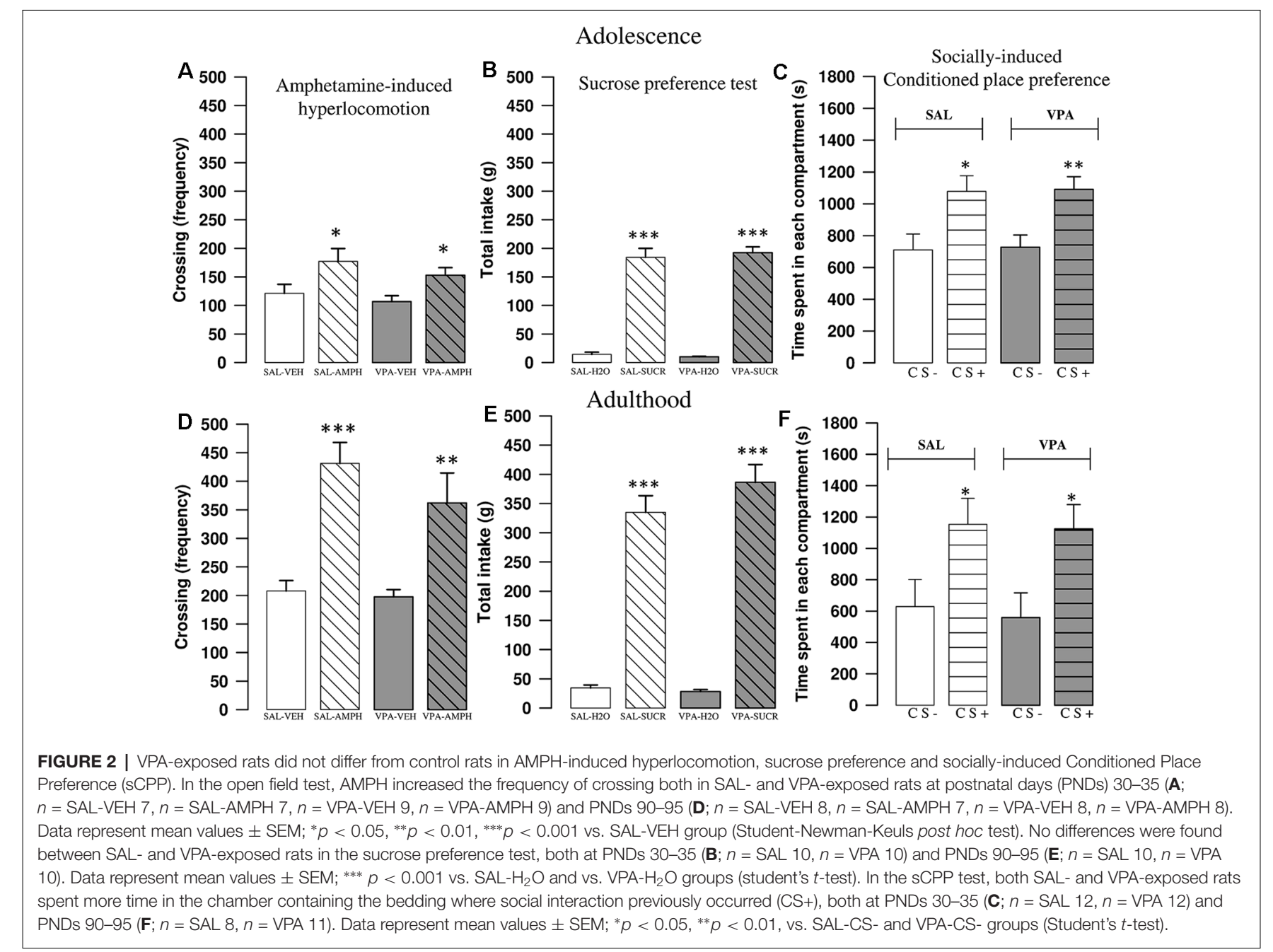

Figure 6A shows representative inward currents obtained in control solution $\left(\mathrm{I}_{\mathrm{aCSF}}\right)$ and $1 \mathrm{mM} \mathrm{Cs}^{+}\left(\mathrm{I}_{\mathrm{Cs}}\right)$. IKir is obtained by subtracting $\mathrm{I}_{\mathrm{Cs}}$ from $\mathrm{I}_{\mathrm{aCSF}}$. Current-voltage relationship reveals a significant reduction of IKir current density in VPA MSNs (SAL MSNs, $n=7$, VPA MSNs, $n=5 ; p<0.05$ where indicated; Figure 6B).

\section{DISCUSSION}

Clinical studies have repeatedly reported that maternal use of VPA during pregnancy can induce a wide range of abnormalities in the exposed children, ranging from structural malformations to more subtle autistic-like behaviors. For this reason, prenatal VPA exposure is nowadays considered an environmental risk factor involved in the pathogenesis of ASD (Christensen et al., 2013; Nicolini and Fahnestock, 2018). Based on the robust clinical evidence, prenatal exposure to VPA in rodents has been validated as a drug-induced preclinical model of ASD (Roullet et al., 2013; Ranger and Ellenbroek, 2016; Tartaglione et al., 2019).
In the present study, we show that prenatal exposure to VPA causes selective deficits in the social domain in the rat offspring at different developmental periods, together with changes in D1 and D2 dopamine receptor expression in the NAc and hyperexcitability in this same brain area, but without inducing changes in social and non-social reward-related behaviors.

According to the DSM-5 diagnostic criteria, persistent deficits in social communication and social interaction are key features of ASD. Social play behavior has a crucial role in the identification and diagnosis of ASD (Jordan, 2003; Jarrold et al., 2013) Social play is the first form of non-mother directed social behavior displayed by most mammals at young age (Panksepp et al., 1984; Vanderschuren et al., 1997, 2016). In ASD, play patterns are characterized not only by deficient cognitive complexity but also by a typical asocial dimension. Since the opportunity to engage in social play is crucial to acquire proper social and cognitive skills (Vanderschuren et al., 2016; Nijhof et al., 2018), the lack of social play in children with ASD has deleterious effects on their development, leading to long lasting deficits in selfawareness, social competence, problem solving and behavioral flexibility. In line with the social deficits reported in previous 


\section{Adolescence}
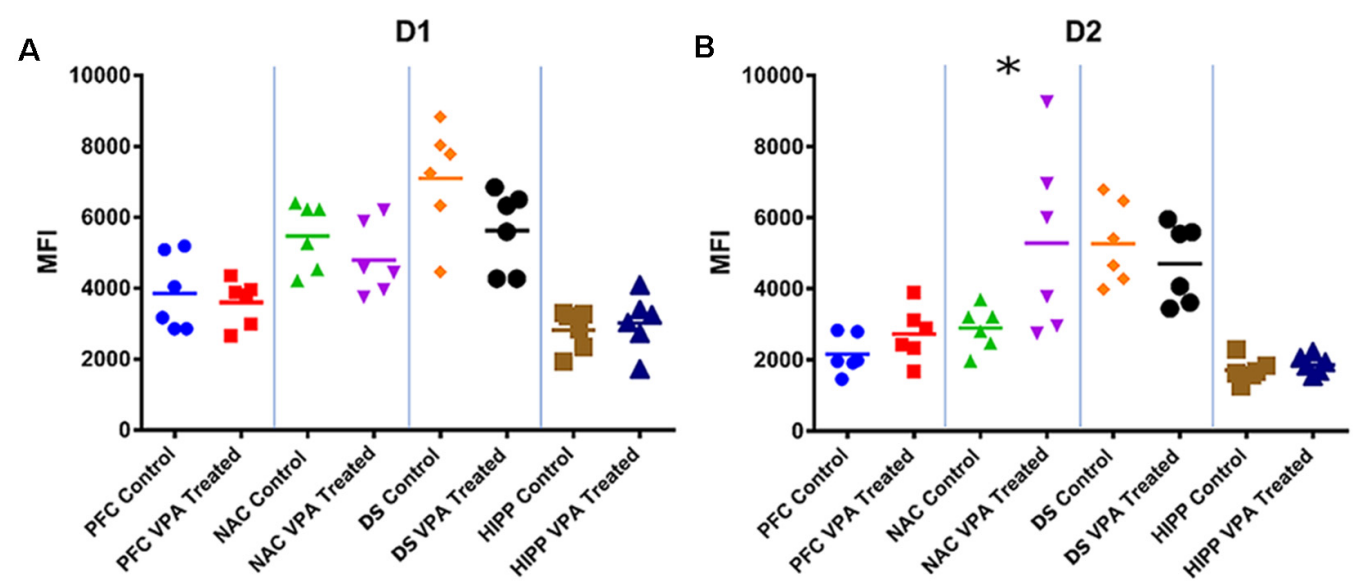

\section{Adulthood}
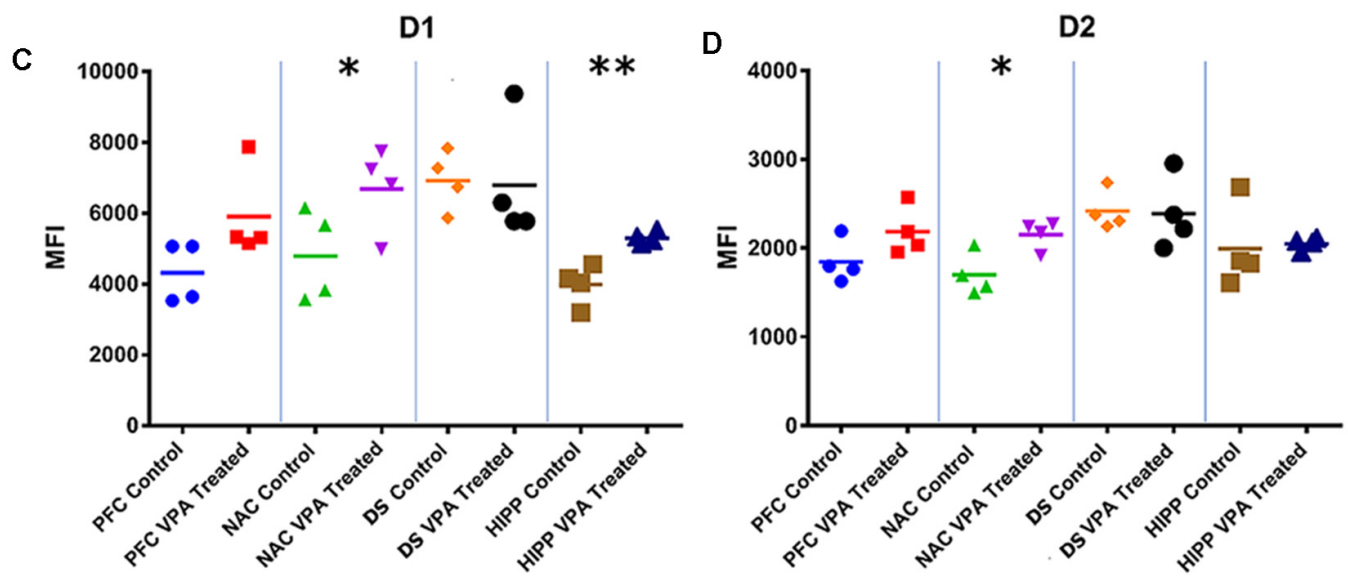

FIGURE 3 | Flow cytometric analysis of dopamine receptors. No differences between VPA-exposed and SAL-exposed rats were found in D1 dopamine receptor expression in prefrontal cortex, nucleus accumbens, dorsal striatum and hippocampus (A). At PNDs 30-35, VPA-exposed rats showed a significant increase in D2 dopamine receptor expression in the nucleus accumbens (NAc) (B) compared to SAL-exposed rats ( $n=$ SAL 6, $n=$ VPA 6). At PNDs 90-95, VPA-exposed rats showed increased expression of D1 dopamine receptor expression in NAc and hippocampus (C), while D2 receptor expression was increased only in the NAc (D; $n=\mathrm{SAL} 5, n=$ VPA 5). Data represent mean values $\pm \mathrm{SEM} ;{ }^{*} p<0.05,{ }^{* *} p<0.01$ vs. SAL group (Student's $t$-test).

studies (Schneider and Przewłocki, 2005; Schneider et al., 2006; Felix-Ortiz and Febo, 2012; Servadio et al., 2016; Melancia et al., 2018), we confirm here that adolescent rats prenatally exposed to VPA show decreased responsiveness to play solicitation, since they respond to play solicitation mainly by partial rotation than reciprocating the playful interaction. Notably, these social deficits were long lasting. Indeed, VPA exposed rats showed social deficits also when tested at adulthood in the three-chamber and social discrimination tests. These data corroborate our previous findings showing that prenatal VPA exposure induces in the rat offspring a wide range of social impairments in the course of development, ranging from social play deficits to altered sociability and social discrimination (Servadio et al., 2016, 2018; Melancia et al., 2018) and are in line with clinical observation that children, adolescents and adults with ASD demonstrate marked socio-communicative deficits (Lai et al., 2011; Dworzynski et al., 2012; Head et al., 2014).

As it has been hypothesized for autistic patients (Chevallier et al., 2012), the wide range of social dysfunctions displayed by VPA-exposed rats may be due to either their inability to properly percept, understand and respond to socially relevant cues, or to a failure of their brain reward system to assign a positive value to the social experience (Pellissier et al., 2018). To address this issue, we performed neurochemical and electrophysiological experiments focusing on brain areas involved in reward processing, and performed additional behavioral experiments to investigate if VPA-exposed rats differ from control animals in responding to social and other (non-social) rewarding stimuli. 
A

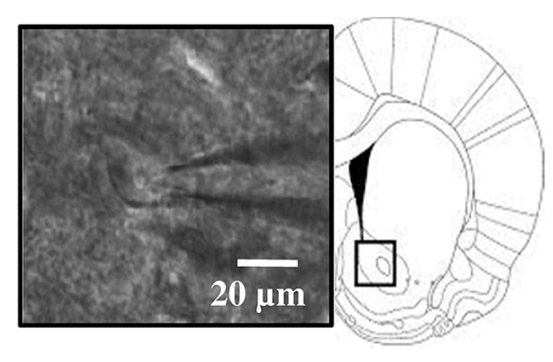

c
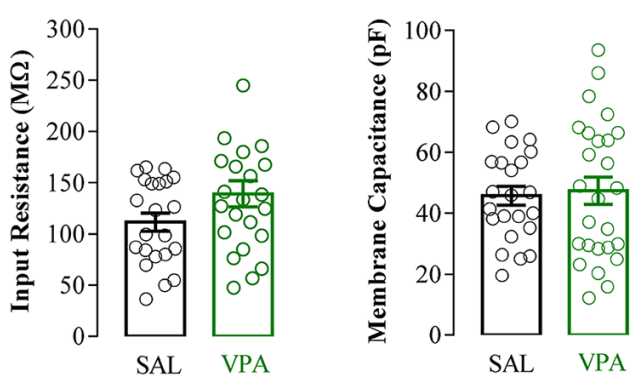

B

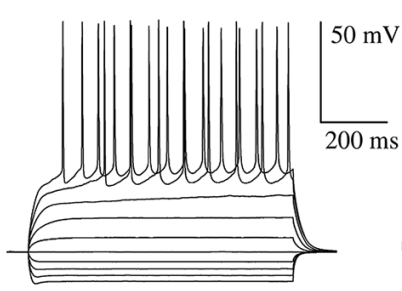

SAL MSNs

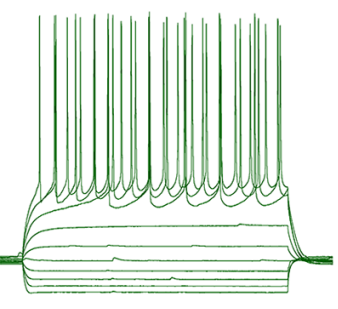

VPA MSNs
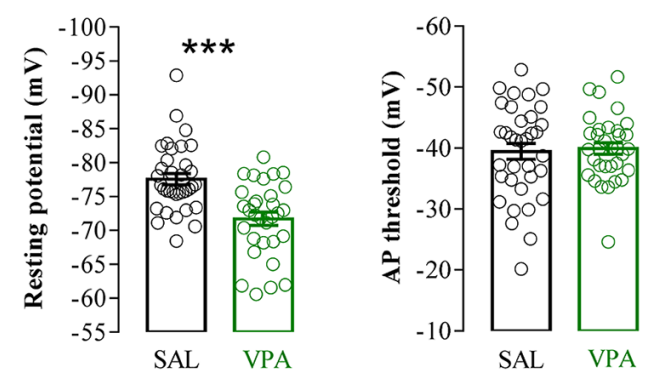

FIGURE 4 | Electrophysiological properties of SAL medium spiny neurons (MSNs) and VPA MSNs. (A) NAc-MSN visualized with infrared video microscopy (left) and schematic representation of a coronal brain slice at the level of the NAc (right). (B) Representative voltage traces of SAL MSNs and VPA MSNs evoked by injection of hyperpolarizing and depolarizing current pulses (800 ms from -200 pA to 250 pA in 50 pA steps). (C) Passive membrane properties of SAL MSNs and VPA MSNs; input resistance (SAL MSNs 111.4 $\pm 8.81 \mathrm{M} \Omega, n=22$; VPA MSNs $139.2 \pm 12.78 \mathrm{M} \Omega, n=23$ ), membrane capacitance (SAL MSNs $45.73 \pm 3.07$ pF $n=23$; VPA MSNs $47.36 \pm 4.49 \mathrm{pF}, n=26$ ); resting membrane potential (SAL MSNs $-77.59 \pm 0.83 \mathrm{mV}, n=35$; VPA MSNs $-71.72 \pm 0.98 \mathrm{mV}, n=37$ ) and AP threshold (SAL MSNs $-39.43 \pm 1.3 \mathrm{mV}, n=35$; VPA MSNs $-39.96 \pm 0.93 \mathrm{mV}, n=33$ ). Data represent mean values $\pm \mathrm{SEM}$; ${ }^{* * *} p<0.001 \mathrm{vs}$. SAL group (Student's $t$-test).

Brain regions involved in the control of social behavior include corticolimbic structures and their altered functionality may represent one neural substrate contributing to the social impairments characteristic of ASD (Scott-Van Zeeland et al., 2010; Chevallier et al., 2012; Ameis and Catani, 2015; Supekar et al., 2018). Notably, these regions are subjected to modulation by dopaminergic neurons and it has been suggested that a dysfunction of dopaminergic neurotransmission in the mesocorticolimbic circuit leads to the social deficits observed in ASD (Paval et al., 2017): thus, it has been demonstrated that striatal MSNs show enriched expression of genes associated with ASD (Chang et al., 2015) and that ASD-associated mutations affect specific MSN subtypes (Portmann et al., 2014; Rothwell et al., 2014; for a review, see Rothwell, 2016).

In autistic subjects, dopamine imbalances, manifested as either hyperactivity or hypoactivity of midbrain dopaminergic pathways, have been detected, highlighting the heterogeneity of the disease (Paval et al., 2017).

Dopamine activation of D1 and D2 receptors in corticolimbic regions is also important for the expression of social behavior in rodents (Robinson et al., 2002; Gunaydin et al., 2014; Manduca et al., 2016; Kopec et al., 2018). Therefore, although the social impairments observed in VPA-exposed animals likely cannot be ascribed to the alteration of a single neurotransmitter system, we focused the biochemical and electrophysiological analyses on dopaminergic neurotransmission in corticolimbic brain areas. We measured the expression of D1 and D2 dopamine receptors in the PFC, DS, NAc and HIPP since these brain areas play not only an important role in the modulation of the rewarding properties of social interactions but have also a key role in cognitive aspects of the social repertoire (e.g., social cognition). While the DS and NAc have a well-recognized role in the modulation of several aspects of reward-related behaviors (for a review, see Bhanji and Delgado, 2014), the PFC and HIPP have also been found to be deeply involved in cognitive aspects of social behavior (Vanderschuren et al., 2016; Montagrin et al., 2018).

We found that, compared to control animals, adolescent rats prenatally exposed to VPA showed increased expression of D2 dopamine receptors in the NAc. At adulthood, they showed increased expression of D1 dopamine receptors in the NAc and hippocampus and of D2 receptors in the NAc. These results suggest that the social deficits displayed by VPA-exposed rats in the course of development may arise from dopaminergic dysfunctions in both these brain regions.

In line with our results, changes in hippocampal dopamine and D1 receptor levels associated with social deficits have been demonstrated in a genetic mouse model exhibiting autism-relevant behavioral abnormalities (Liu et al., 2017). Furthermore, mice knockout for genes strongly associated with ASD, such as Cntnap4 (Karayannis et al., 2014) and neuroligin-3 (Rothwell et al., 2014) mutant mice, show changes in NAc dopaminergic neurotransmission together 


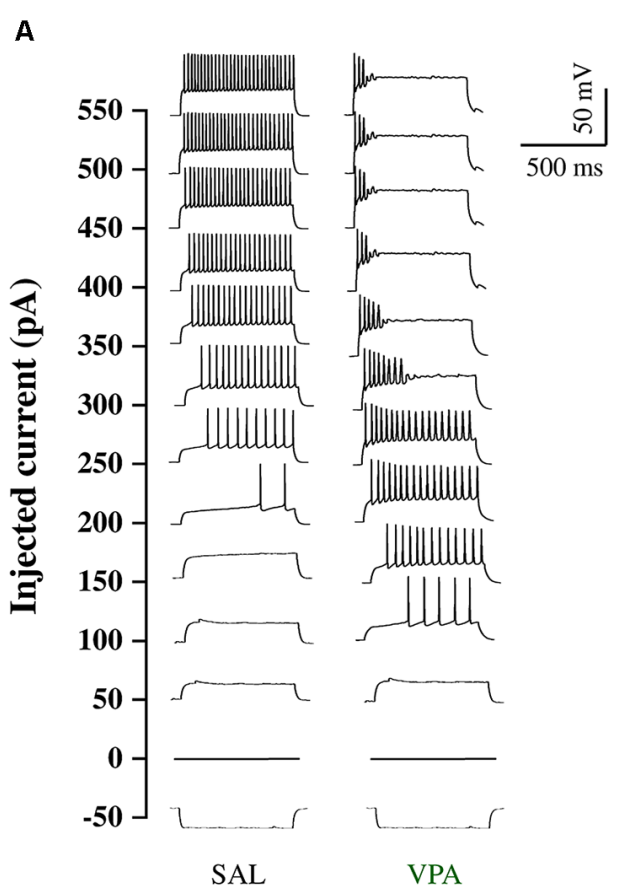

B

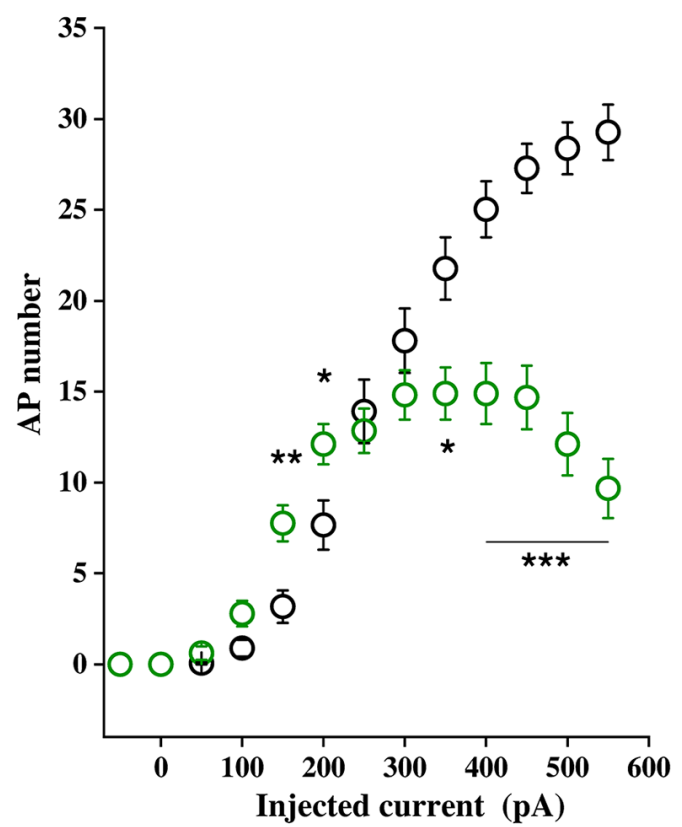

FIGURE 5 | Intrinsic excitability of SAL MSNs and VPA MSNs. (A) Representative voltage traces obtained in response to-50 to 550 pA current steps (800 ms duration, 50 pA amplitude) from resting potential, recorded from SAL and VPA MSNs. (B) Number of APs fired plotted against current steps of increasing value (SAL MSNs, $n=35$; VPA MSNs, $n=37$ ). Data represent mean values \pm SEM; ${ }^{*} p<0.05,{ }^{* *} p<0.01,{ }^{* * *} p<0.001$ vs. SAL group (Student's $t$-test).

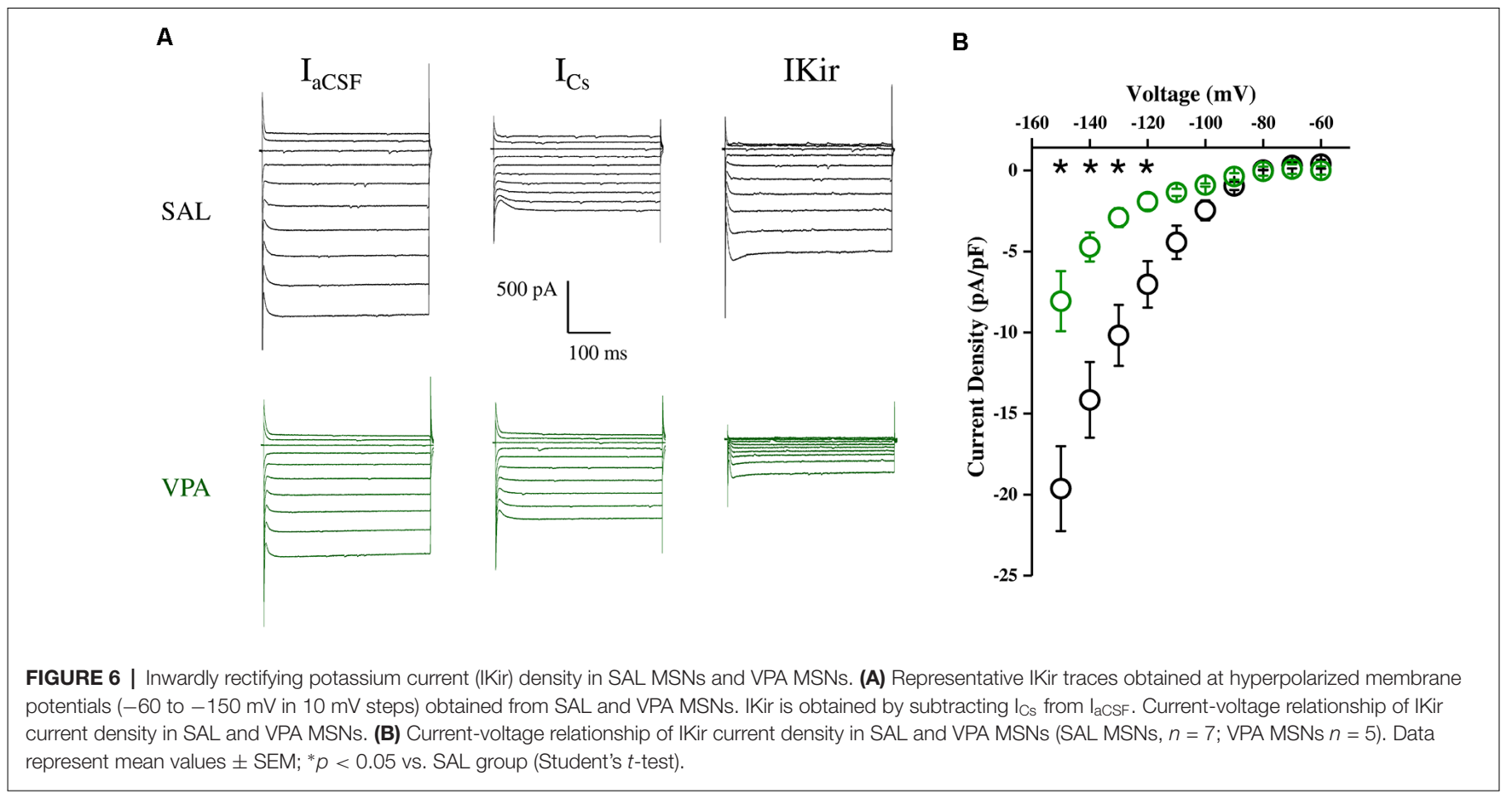

with deficits in the core ASD behavioral domains. Recently, it has also been demonstrated that altered VTA dopamine neuron function represents a key mechanism by which insufficiency of SHANK3, encoding the synapse scaffolding protein SHANK3, generates impaired social preference in mice (Bariselli et al., 2016). 
Building on this evidence, we studied the basic electrophysiological properties of NAc MSNs in acute striatal brain slices of VPA- and SAL-exposed offspring. We found that NAc MSNs of VPA-exposed animals show a significant depolarization of the resting membrane potential and increased excitability in the lower part of the excitability curve, indicating higher firing probability in conditions of normal synaptic excitation. These changes are likely caused by altered Kir current density, known to determine the hyperpolarized value of membrane potential in normal MSNs $(\sim-85 \mathrm{mV})$ and to affect their AP discharge pattern (Kreitzer, 2009; Cazorla et al., 2012). The NAc is a major node of the mesolimbic dopaminergic system and the overall impact of NAc output on behavior depends on the relative activity of D1- vs. D2-expressing MSNs. Increased excitability of MSNs of VPA-exposed animals, combined with increased expression of D2R-expressing neurons, is consistent with an imbalance in the direct and indirect pathway activity, in favor of the latter. Proper activation of these pathways underlies proper motor learning as well as the acquisition of reward-related behaviors (Yawata et al., 2012; Shin et al., 2018). Furthermore, extensive alterations in normal gene expression pattern have been found in multiple striatal neuronal populations in the VPA model of ASD (Lauber et al., 2016).

Based on the evidence of neurochemical and electrophysiological changes in the brain reward system of VPA-exposed rats, we tested their behavioral response to different (i.e., social and non-social) rewarding stimuli. First, we tested whether VPA- and SAL-exposed rats differently responded to a dose of amphetamine known to induce hyperlocomotion (Bolanos et al., 1998), a proxy for the ability of the dopaminergic system to respond to pharmacological activation. We found a robust amphetamine-induced increase in motor activity in the open field test in both adolescent and adult VPA- and SAL-exposed animals, showing a same susceptibility to amphetamine-induced hyperlocomotion in both experimental groups.

To examine whether the social impairment displayed by VPA-exposed rats was accompanied by a generalized anhedonic behavior, that is, a reduction in the interest for natural reward, we performed a sucrose preference test. In line with previous studies performed with other preclinical models of ASD (Jamain et al., 2008; Radyushkin et al., 2009) and with clinical studies reporting intact hedonic responses to sweet taste in autistic patients (Damiano et al., 2014), we found that both VPA-exposed and control animals showed preference for the sucrose over the water solution, either at adolescence or adulthood.

To assess whether the social deficits displayed by VPA-exposed animals may be due to aberrant social reward processing, we tested VPA- and SAL-exposed rats in a sociallyinduced place-conditioning task (sCPP), which involves the association between a social stimulus and a distinct set of contextual cues (Trezza and Vanderschuren, 2009; Dölen et al., 2013; Wei et al., 2015). Attenuated sCPP has been demonstrated in genetic models of ASD such as in fmr1 mutant mice, a model for Fragile $\mathrm{X}$ syndrome (Pacey et al., 2011), and BTBR T+tf/J (BTBR) mice (Pearson et al., 2012). However, we here failed to find any difference in sCPP: thus, both VPA- and SAL-exposed animals spent more time during testing in the chamber associated with a bedding where social interaction previously occurred. Thus, the altered pattern of social play behavior, the reduced sociability and the impaired social discrimination abilities displayed by VPA-exposed rats are not accompanied by altered social reward processing.

\section{LIMITATIONS}

From the behavioral point of view, the present study has some methodological limitations. Indeed, while we here found that VPA- and SAL-exposed rats similarly respond to amphetamineinduced hyperlocomotion, it still needs to be determined whether VPA- and SAL-exposed animals differ in behavioral set-ups specifically designed to assess drug intake and drug addiction. Similarly, we cannot exclude that VPA-exposed animals would show altered sucrose preference if different concentrations of sucrose solution were used, as found in the SHANK3 mouse model of ASD, where Shank3 mice preferred a sucrose solution at high but not at low concentrations (Bariselli et al., 2016). Last, it is still possible that VPA-exposed rats would show altered social motivation when tested in socially-driven operant conditioning paradigms (Achterberg et al., 2016). Concerning the electrophysiological experiments, the potential limitations in the validity of the information obtained with brain slice recordings is related to the nature of the methodology, which allows for in-depth, reliable interrogation of cell-autonomous electrical properties, but inevitably involves profound alteration of network connectivity, even within the area under examination. Aware of the intrinsic advantages and limitations of this approach, here we focused on neuronal properties that are better preserved in the acute brain slice preparation, such as intrinsic neuronal excitability.

\section{CONCLUSION}

Although more levels of analysis are needed to shed light on the mechanisms underlying the aberrant behavior found in VPA-exposed rats, on the basis of the results obtained here we suggest that the reduced play responsiveness, the impaired sociability in the three-chamber test and the reduced discrimination abilities in the social discrimination tasks displayed by VPA-exposed animals reported in the present and in previous studies (Kim et al., 2011, 2013, 2014; Servadio et al., 2016; Melancia et al., 2018) are more likely due to changes in the cognitive functions required for proper social interaction, such as to understand and predict the behaviors of other conspecifics or to adapt the social behaviors of the animal to the changing circumstances in its social and physical environment, or may be due to impairments in aspects of reward processing that could not be detected with the behavioral tasks used in the present work. In this picture, the alteration in the expression of striatal dopamine receptors and in the electrical 
properties of MSNs in the NAc may be interpreted as a homeostatic mechanism deployed by reward-related brain areas to compensate for VPA-induced neurodevelopmental perturbations. Further behavioral, neurochemical and electrophysiological investigations are required to support this interpretation.

\section{DATA AVAILABILITY STATEMENT}

The datasets used and/or analyzed during the current study are available from the corresponding author on reasonable request.

\section{ETHICS STATEMENT}

The experiments were approved by the Italian Ministry of Health (Rome, Italy) and performed in agreement with the ARRIVE (Animals in Research: Reporting in vivo Experiments; Kilkenny et al., 2010) guidelines, with the guidelines released by the Italian Ministry of Health (D.L. 26/14) and the European Community Directive 2010/63/EU. In particular, the experimental protocol was approved by the Animal Care Committees of both Roma Tre and Florence Universities and by the Italian Ministry of Health (authorization numbers: 31-2019-PR and 955/2015-PR).

\section{REFERENCES}

Achterberg, E. J., Van Kerkhof, L. W., Servadio, M., Van Swieten, M. M., Houwing, D. J., Aalderink, M., et al. (2016). Contrasting roles of dopamine and noradrenaline in the motivational properties of social play behavior in rats. Neuropsychopharmacology 41, 858-868. doi: 10.1038/npp. 2015.212

Ameis, S. H., and Catani, M. (2015). Altered white matter connectivity as a neural substrate for social impairment in autism spectrum disorder. Cortex 62, 158-181. doi: 10.1016/j.cortex.2014.10.014

Bariselli, S., Tzanoulinou, S., Glangetas, C., Prevost-Solie, C., Pucci, L., Viguie, J., et al. (2016). SHANK3 controls maturation of social reward circuits in the VTA. Nat. Neurosci. 19, 926-934. doi: 10.1038/nn.4319

Bhanji, J. P., and Delgado, M. R. (2014). The social brain and reward: social information processing in the human striatum. Wiley Interdiscip. Rev. Cogn. Sci. 5, 61-73. doi: 10.1002/wcs. 1266

Bolanos, C. A., Glatt, S. J., and Jackson, D. (1998). Subsensitivity to dopaminergic drugs in periadolescent rats: a behavioral and neurochemical analysis. Dev. Brain Res. 111, 25-33. doi: 10.1016/s0165-3806(98)00116-3

Bronzuoli, M. R., Facchinetti, R., Ingrassia, D., Sarvadio, M., Schiavi, S., Steardo, L., et al. (2018). Neuroglia in the autistic brain: evidence from a preclinical model. Mol. Autism 9:66. doi: 10.1186/s13229-018-0254-0

Campolongo, P., Trezza, V., Cassano, T., Gaetani, S., Morgese, M. G., Ubaldi, M., et al. (2007). Perinatal exposure to delta-9-tetrahydrocannabinol causes enduring cognitive deficits associated with alteration of cortical gene expression and neurotransmission in rats. Addict. Biol. 12, 485-495. doi: 10.1111/j.1369-1600.2007.00074.x

Carbone, C., Costa, A., Provensi, G., Mannaioni, G., and Masi, A. (2017). The hyperpolarization-activated current determines synaptic excitability, calcium activity and specific viability of substantia nigra dopaminergic neurons. Front. Cell. Neurosci. 11:187. doi: 10.3389/fncel.2017.00187

Cartocci, V., Catallo, M., Tempestilli, M., Segatto, M., Pfrieger, F. W., Bronzuoli, M. R., et al. (2018). Altered brain cholesterol/isoprenoid metabolism

\section{AUTHOR CONTRIBUTIONS}

SS and FM performed, analyzed and contributed to the design of the behavioral experiments. SL performed, analyzed and designed the flow cytometric experiments. DI, CC and MP performed, analyzed and contributed to the design of the electrophysiology experiments. AMan and GM contributed to the design of the experiments and edited the manuscript. SS and DI wrote the manuscript. VT and AMas supervised the project, designed the experiments and wrote the manuscript.

\section{FUNDING}

This work was supported by Marie Curie Career Reintegration Grant PCIG09-GA-2011-293589 (VT), by Jerome Lejeune Foundation (Fondation Jérôme Lejeune) Research grant \#1674 (VT) and by Excellence Departments, Ministero dell'Istruzione, dell'Università e della Ricerca (MIUR)-Italy, Grant/Award Numbers: ARTICOLO 1, COMMI 314- 337 LEGGE 232/2016, ARTICOLO 1.

\section{ACKNOWLEDGMENTS}

We thank and gratefully acknowledge Mrs. Claudia Sereni, Patricia Jiménez Peinado and Giulia Polese for technical help during the experiments.

in a rat model of autism spectrum disorders. Neuroscience 372, 27-37. doi: 10.1016/j.neuroscience.2017.12.053

Cazorla, M., Shegda, M., Ramesh, B., Harrison, N. L., and Kellendonk, C. (2012) Striatal D2 receptors regulate dendritic morphology of medium spiny neurons via Kir2 channels. J. Neurosci. 32, 2398-2409. doi: 10.1523/JNEUROSCI.605611.2012

Cepeda, C., André, V. M., Yamazaki, I., Wu, N., Kleiman-Weiner, M., and Levine, M. S. (2008). Differential electrophysiological properties of dopamine D1 and D2 receptor-containing striatal medium-sized spiny neurons. Eur. J. Neurosci. 27, 671-682. doi: 10.1111/j.1460-9568.2008. 06038.x

Chang, J., Gilman, S. R., Chiang, A. H., Sanders, S. J., and Vitkup, D. (2015). Genotype to phenotype relationships in autism spectrum disorders. Nat. Neurosci. 18, 191-198. doi: 10.1038/nn.3907

Chevallier, C., Kohls, G., Troiani, V., Brodkin, E. S., and Schultz, R. T. (2012). The social motivation theory of autism. Trends Cogn. Sci. 16, 231-239. doi: 10.1016/j.tics.2012.02.007

Christensen, J., Grønborg, T. K., Sørensen, M. J., Schendel, D., Parner, E. T., Pedersen, L. H., et al. (2013). Prenatal valproate exposure and risk of autism spectrum disorders and childhood autism. JAMA 309, 1696-1703. doi: 10.1001/jama.2013.2270

Cruz, F. C., Koya, E., Guez-Barber, D. H., Bossert, J. M., Lupica, C. R., Shaham, Y., et al. (2013). New technologies for examining the role of neuronal ensembles in drug addiction and fear. Nat. reviews. Neurosci. 14, 743-754. doi: $10.1038 / \mathrm{nrn} 3597$

Damiano, C. R., Aloi, J., Burrus, C., Garbutt, J. C., Kampov-Polevoy, A. B., and Dichter, G. S. (2014). Intact hedonic responses to sweet tastes in autism spectrum disorder. Res. Autism Spectr. Disord. 8, 230-236. doi: 10.1016/j.rasd. 2013.12.003

Dölen, G., Darvishzadeh, A., Huang, K. W., and Malenka, R. C. (2013). Social reward requires coordinated activity of nucleus accumbens oxytocin and serotonin. Nature 501, 179-184. doi: 10.1038/nature 12518 
Dufour-Rainfray, D., Vourc'h, P., Le Guisquet, A. M., Garreau, L., Ternant, D., Bodard, S., et al. (2010). Behavior and serotonergic disorders in rats exposed prenatally to valproate: a model for autism. Neurosci. Lett. 470, 55-59. doi: 10.1016/j.neulet.2009.12.054

Dworzynski, K., Ronald, A., Bolton, P., and Happe, F. (2012). How different are girls and boys above and below the diagnostic threshold for autism spectrum disorders? J. Am. Acad. Child Adolesc. Psychiatry 51, 788-797. doi: 10.1016/j. jaac.2012.05.018

Favre, M. R., Barkat, T. R., Lamendola, D., Khazen, G., Markram, H., and Markram, K. (2013). General developmental health in the VPA-rat model of autism. Front. Behav. Neurosci. 7:88. doi: 10.3389/fnbeh.2013. 00088

Felix-Ortiz, A. C., and Febo, M. (2012). Gestational valproate alters BOLD activation in response to complex social and primary sensory stimuli. PLoS One 7:e37313. doi: 10.1371/journal.pone.0037313

Fontes-Dutra, M., Della-Flora Nunes, G., Santos-Terra, J., Souza-Nunes, W., Bauer-Negrini, G., Hirsch, M. M., et al. (2019). Abnormal empathy-like pro-social behaviour in the valproic acid model of autism spectrum disorder. Behav. Brain Res. 364, 11-18. doi: 10.1016/j.bbr.2019.01.034

Fowler, S. C., Birkestrand, B., Chen, R., Vorontsova, E., and Zarcone, T. (2003). Behavioral sensitization to amphetamine in rats: changes in the rhythm of head movements during focused stereotypies. Psychopharmacology 170, 167-177. doi: 10.1007/s00213-003-1528-5

Gray, J. M., Vecchiarelli, H. A., Morena, M., Lee, T. T., Hermanson, D. J., Kim, A. B., et al. (2015). Corticotropin-releasing hormone drives anandamide hydrolysis in the amygdala to promote anxiety. J. Neurosci. 35, 3879-3892. doi: 10.1523/JNEUROSCI.2737-14.2015

Gunaydin, L. A., Grosenick, L., Finkelstein, J. C., Kauvar, I. V., Fenno, L. E., Adhikari, A., et al. (2014). Natural neural projection dynamics underlying social behavior. Cell 157, 1535-1551. doi: 10.1016/j.cell.2014.05.017

Head, A. M., McGillivray, J. A., and Stokes, M. A. (2014). Gender differences in emotionality and sociability in children with autism spectrum disorders. Mol. Autism 5:19. doi: 10.1186/2040-2392-5-19

Hill, M. N., Karatsoreos, I. N., Hillard, C. J., and Mcewen, B. S. (2010). Rapid elevations in limbic endocannabinoid content by glucocorticoid hormones in vivo. Psychoneuroendocrinology 35, 1333-1338. doi: 10.1016/j.psyneuen. 2010.03.005

Hirsch, M. M., Deckmann, I., Fontes-Dutra, M., Bauer-Negrini, G., Della-Flora Nunes, G., Nunes, W., et al. (2018). Behavioral alterations in autism model induced by valproic acid and translational analysis of circulating microRNA. Food Chem. Toxicol. 115, 336-343. doi: 10.1016/j.fct.2018.02.061

Jamain, S., Radyushkin, K., Hammerschmidt, K., Granon, S., Boretius, S., Varoqueaux, F., et al. (2008). Reduced social interaction and ultrasonic communication in a mouse model of monogenic heritable autism. Proc. Natl. Acad. Sci. U S A 105, 1710-1715. doi: 10.1073/pnas.0711555105

Jarrold, W., Mundy, P., Gwaltney, M., Bailenson, J., Hatt, N., Mcintyre, N., et al. (2013). Social attention in a virtual public speaking task in higher functioning children with autism. Autism Res. 6, 393-410. doi: 10.1002/aur.1302

Jordan, R. (2003). Social play and autistic spectrum disorders: a perspective on theory, implications and educational approaches. Autism 7, 347-360. doi: $10.1177 / 1362361303007004002$

Karayannis, T., Au, E., Patel, J. C., Kruglikov, I., Markx, S., Delorme, R., et al. (2014). Cntnap4 differentially contributes to GABAergic and dopaminergic synaptic transmission. Nature 511, 236-240. doi: 10.1038/nature13248

Kilkenny, C., Browne, W. J., Cuthill, I. C., Emerson, M., and Altman, D. G. (2010). Improving bioscience research reporting: the ARRIVE guidelines for reporting animal research. J. Pharmacol. Pharmacother. 1, 94-99. doi: 10.4103/0976500X.72351

Kim, K. C., Kim, P., Go, H. S., Choi, C. S., Park, J. H., Kim, H. J., et al. (2013). Male-specific alteration in excitatory post-synaptic development and social interaction in pre-natal valproic acid exposure model of autism spectrum disorder. J. Neurochem. 124, 832-843. doi: 10.1111/jnc.12147

Kim, K. C., Kim, P., Go, H. S., Choi, C. S., Yang, S. I., Cheong, J. H., et al. (2011). The critical period of valproate exposure to induce autistic symptoms in Sprague-Dawley rats. Toxicol. Lett. 201, 137-142. doi: 10.1016/j.toxlet.2010. 12.018

Kim, K. C., Lee, D. K., Go, H. S., Kim, P., Choi, C. S., Kim, J. W., et al. (2014). Pax6dependent cortical glutamatergic neuronal differentiation regulates autism-like behavior in prenatally valproic acid-exposed rat offspring. Mol. Neurobiol. 49, 512-528. doi: 10.1007/s12035-013-8535-2

Kini, U., Adab, N., Vinten, J., Fryer, A., and Clayton-Smith, J. (2006). Dysmorphic features: an important clue to the diagnosis and severity of fetal anticonvulsant syndromes. Arch. Dis. Child Fetal Neonatal. Ed. 91, F90-F95. doi: 10.1136/adc. 2004.067421

Kopec, A. M., Smith, C. J., Ayre, N. R., Sweat, S. C., and Bilbo, S. D. (2018). Microglial dopamine receptor elimination defines sex-specific nucleus accumbens development and social behavior in adolescent rats. Nat. Commun. 9:3769. doi: 10.1038/s41467-018-06118-z

Kozma, C. (2001). Valproic acid embryopathy: report of two siblings with further expansion of the phenotypic abnormalities and a review of the literature. Am. J. Med. Genet. 98, 168-175. doi: 10.1002/10968628(20010115)98:2\%3C168::AID-AJMG1026\%3E3.0.CO;2-O

Kreitzer, A. C. (2009). Physiology and pharmacology of striatal neurons. Annu. Rev. Neurosci. 32, 127-147. doi: 10.1146/annurev.neuro.051508.135422

Lai, M. C., Lombardo, M. V., Pasco, G., Ruigrok, A. N., Wheelwright, S. J., Sadek, S. A., et al. (2011). A behavioral comparison of male and female adults with high functioning autism spectrum conditions. PLoS One 6:e20835. doi: 10.1371/journal.pone.0020835

Lauber, E., Filice, F., and Schwaller, B. (2016). Prenatal valproate exposure differentially affects parvalbumin-expressing neurons and related circuits in the cortex and striatum of mice. Front. Mol. Neurosci. 9:150. doi: 10.3389/fnmol. 2016.00150

Liu, Q., Shi, J., Lin, R., and Wen, T. (2017). Dopamine and dopamine receptor D1 associated with decreased social interaction. Behav. Brain Res. 324, 51-57. doi: 10.1016/j.bbr.2017.01.045

Manduca, A., Lassalle, O., Sepers, M., Campolongo, P., Cuomo, V., Marsicano, G., et al. (2016). Interacting cannabinoid and opioid receptors in the nucleus accumbens core control adolescent social play. Front. Behav. Neurosci. 10:211. doi: 10.3389/fnbeh.2016.00211

Manduca, A., Servadio, M., Campolongo, P., Palmery, M., Trabace, L., Vanderschuren, L. J., et al. (2014). Strain- and context-dependent effects of the anandamide hydrolysis inhibitor URB597 on social behavior in rats Eur. Neuropsychopharmacol. 24, 1337-1348. doi: 10.1016/j.euroneuro.2014. 05.009

Markram, K., Rinaldi, T., La Mendola, D., Sandi, C., and Markram, H. (2008). Abnormal fear conditioning and amygdala processing in an animal model of autism. Neuropsychopharmacology 33, 901-912. doi: 10.1038/sj.npp. 1301453

Melancia, F., Schiavi, S., Servadio, M., Cartocci, V., Campolongo, P., Palmery, M., et al. (2018). Sex-specific autistic endophenotypes induced by prenatal exposure to valproic acid involve anandamide signalling. Br. J. Pharmacol. 175, 3699-3712. doi: 10.1111/bph.14435

Miyazaki, K., Narita, N., and Narita, M. (2005). Maternal administration of thalidomide or valproic acid causes abnormal serotonergic neurons in the offspring: implication for pathogenesis of autism. Int. J. Dev. Neurosci. 23, 287-297. doi: 10.1016/j.ijdevneu.2004.05.004

Montagrin, A., Saiote, C., and Schiller, D. (2018). The social hippocampus. Hippocampus 28, 672-679. doi: 10.1002/hipo.22797

Monteggia, L. M., Luikart, B., Barrot, M., Theobold, D., Malkovska, I., Nef, S., et al. (2007). Brain-derived neurotrophic factor conditional knockouts show gender differences in depression-related behaviors. Biol. Psychiatry 61, 187-197. doi: 10.1016/j.biopsych.2006.03.021

Narita, N., Kato, M., Tazoe, M., Miyazaki, K., Narita, M., and Okado, N. (2002). Increased monoamine concentration in the brain and blood of fetal thalidomide- and valproic acid-exposed rat: putative animal models for autism. Pediatr. Res. 52, 576-579. doi: 10.1203/00006450-20021000000018

Nicolini, C., and Fahnestock, M. (2018). The valproic acid-induced rodent model of autism. Exp. Neurol. 299, 217-227. doi: 10.1016/j.expneurol.2017. 04.017

Nijhof, S. L., Vinkers, C. H., Van Geelen, S. M., Duijff, S. N., Achterberg, E. J. M., Van Der Net, J., et al. (2018). Healthy play, better coping: the importance of play for the development of children in health and disease. Neurosci. Biobehav. Rev. 95, 421-429. doi: 10.1016/j.neubiorev.2018.09.024

Pacey, L. K., Doss, L., Cifelli, C., Van Der Kooy, D., Heximer, S. P., and Hampson, D. R. (2011). Genetic deletion of regulator of G-protein signaling 4 
(RGS4) rescues a subset of fragile X related phenotypes in the FMR1 knockout mouse. Mol. Cell. Neurosci. 46, 563-572. doi: 10.1016/j.mcn.2010.12.005

Panksepp, J., Siviy, S., and Normansell, L. (1984). The psychobiology of play: theoretical and methodological perspectives. Neurosci. Biobehav. Rev. 8, 465-492. doi: 10.1016/0149-7634(84)90005-8

Paval, D., Rad, F., Rusu, R., Niculae, A. S., Colosi, H. A., Dobrescu, I., et al. (2017). Low Retinal Dehydrogenase 1 (RALDH1) level in prepubertal boys with autism spectrum disorder: a possible link to dopamine dysfunction? Clin. Psychopharmacol. Neurosci. 15, 229-236. doi: 10.9758/cpn.2017.15.3.229

Pearson, B. L., Bettis, J. K., Meyza, K. Z., Yamamoto, L. Y., Blanchard, D. C., and Blanchard, R. J. (2012). Absence of social conditioned place preference in BTBR $\mathrm{T}+\mathrm{tf} / \mathrm{J}$ mice: relevance for social motivation testing in rodent models of autism. Behav. Brain Res. 233, 99-104. doi: 10.1016/j.bbr.2012.04.040

Pellissier, L. P., Gandia, J., Laboute, T., Becker, J. A. J., and Le Merrer, J. (2018). $\mu$-opioid receptor, social behaviour and autism spectrum disorder: reward matters. Br. J. Pharmacol. 175, 2750-2769. doi: 10.1111/bph.13808

Portmann, T., Yang, M., Mao, R., Panagiotakos, G., Ellegood, J., Dolen, G., et al. (2014). Behavioral abnormalities and circuit defects in the basal ganglia of a mouse model of 16p11.2 deletion syndrome. Cell Rep. 7, 1077-1092. doi: 10.1016/j.celrep.2014.03.036

Radyushkin, K., Hammerschmidt, K., Boretius, S., Varoqueaux, F., El-Kordi, A., Ronnenberg, A., et al. (2009). Neuroligin-3-deficient mice: model of a monogenic heritable form of autism with an olfactory deficit. Genes Brain Behav. 8, 416-425. doi: 10.1111/j.1601-183X.2009.00487.X

Ranger, P., and Ellenbroek, B. A. (2016). Perinatal influences of valproate on brain and behaviour: an animal model for autism. Curr. Top. Behav. Neurosci. 29, 363-386. doi: 10.1007/7854_2015_404

Ricceri, L., Moles, A., and Crawley, J. (2007). Behavioral phenotyping of mouse models of neurodevelopmental disorders: relevant social behavior patterns across the life span. Behav. Brain Res. 176, 40-52. doi: 10.1016/j.bbr.2006. 08.024

Robinson, D. L., Heien, M. L., and Wightman, R. M. (2002). Frequency of dopamine concentration transients increases in dorsal and ventral striatum of male rats during introduction of conspecifics. J. Neurosci. 22, 10477-10486. doi: 10.1523/jneurosci.22-23-10477.2002

Rodier, P. M., Ingram, J. L., Tisdale, B., Nelson, S., and Romano, J. (1996). Embryological origin for autism: developmental anomalies of the cranial nerve motor nuclei. J. Comp. Neurol. 370, 247-261. doi: 10.1002/(sici)10969861(19960624)370:2<247::aid-cne8>3.0.c0;2-2

Rothwell, P. E. (2016). Autism spectrum disorders and drug addiction: common pathways, common molecules, distinct disorders? Front. Neurosci. 10:20. doi: 10.3389/fnins.2016.00020

Rothwell, P. E., Fuccillo, M. V., Maxeiner, S., Hayton, S. J., Gokce, O., Lim, B. K., et al. (2014). Autism-associated neuroligin-3 mutations commonly impair striatal circuits to boost repetitive behaviors. Cell 158, 198-212. doi: 10.1016/j. cell.2014.04.045

Roullet, F. I., Lai, J. K., and Foster, J. A. (2013). In utero exposure to valproic acid and autism-a current review of clinical and animal studies. Neurotoxicol. Teratol. 36, 47-56. doi: 10.1016/j.ntt.2013.01.004

Schneider, T., and Przewłocki, R. (2005). Behavioral alterations in rats prenatally exposed to valproic acid: animal model of autism. Neuropsychopharmacology 30, 80-89. doi: 10.1038/sj.npp.1300518

Schneider, T., Roman, A., Basta-Kaim, A., Kubera, M., Budziszewska, B., Schneider, K., et al. (2008). Gender-specific behavioral and immunological alterations in an animal model of autism induced by prenatal exposure to valproic acid. Psychoneuroendocrinology 33, 728-740. doi: 10.1016/j.psyneuen. 2008.02.011

Schneider, T., Turczak, J., and Przewłocki, R. (2006). Environmental enrichment reverses behavioral alterations in rats prenatally exposed to valproic acid: issues for a therapeutic approach in autism. Neuropsychopharmacology 31, 36-46. doi: 10.1038/sj.npp.1300767

Scott-Van Zeeland, A. A., Dapretto, M., Ghahremani, D. G., Poldrack, R. A., and Bookheimer, S. Y. (2010). Reward processing in autism. Autism Res. 3, 53-67. doi: 10.1002/aur.122
Servadio, M., Manduca, A., Melancia, F., Leboffe, L., Schiavi, S., Campolongo, P., et al. (2018). Impaired repair of DNA damage is associated with autistic-like traits in rats prenatally exposed to valproic acid. Eur. Neuropsychopharmacol. 28, 85-96. doi: 10.1016/j.euroneuro.2017.11.014

Servadio, M., Melancia, F., Manduca, A., Di Masi, A., Schiavi, S., Cartocci, V., et al. (2016). Targeting anandamide metabolism rescues core and associated autistic-like symptoms in rats prenatally exposed to valproic acid. Transl. Psychiatry 6:e902. doi: 10.1038/tp.2016.182

Shin, J. H., Kim, D., and Jung, M. W. (2018). Differential coding of reward and movement information in the dorsomedial striatal direct and indirect pathways. Nat. Commun. 9:404. doi: 10.1038/s41467-017-02817-1

Supekar, K., Kochalka, J., Schaer, M., Wakeman, H., Qin, S., Padmanabhan, A., et al. (2018). Deficits in mesolimbic reward pathway underlie social interaction impairments in children with autism. Brain 141, 2795-2805. doi: 10.1093/brain/awy191

Tartaglione, A. M., Schiavi, S., Calamandrei, G., and Trezza, V. (2019). Prenatal valproate in rodents as a tool to understand the neural underpinnings of social dysfunctions in autism spectrum disorder. Neuropharmacology. doi: 10.1016/j. neuropharm.2018.12.024 [Epub ahead of print].

Trezza, V., and Vanderschuren, L. J. (2008). Bidirectional cannabinoid modulation of social behavior in adolescent rats. Psychopharmacology 197, 217-227. doi: 10.1007/s00213-007-1025-3

Trezza, V., and Vanderschuren, L. J. (2009). Divergent effects of anandamide transporter inhibitors with different target selectivity on social play behavior in adolescent rats. J. Pharmacol. Exp. Ther. 328, 343-350. doi: 10.1124/jpet.108. 141069

Trezza, V., Damsteegt, R., Manduca, A., Petrosino, S., Van Kerkhof, L. W., Pasterkamp, R. J., et al. (2012). Endocannabinoids in amygdala and nucleus accumbens mediate social play reward in adolescent rats. J. Neurosci. 32, 14899-14908. doi: 10.1523/JNEUROSCI.0114 $-12.2012$

Vanderschuren, L. J., Achterberg, E. J., and Trezza, V. (2016). The neurobiology of social play and its rewarding value in rats. Neurosci. Biobehav. Rev. 70, 86-105. doi: 10.1016/j.neubiorev.2016.07.025

Vanderschuren, L. J., Niesink, R. J., and Van Ree, J. M. (1997). The neurobiology of social play behavior in rats. Neurosci. Biobehav. Rev. 21, 309-326. doi: 10.1016/s0149-7634(96)00020-6

Wei, D., Lee, D., Cox, C. D., Karsten, C. A., Penagarikano, O., Geschwind, D. H., et al. (2015). Endocannabinoid signaling mediates oxytocin-driven social reward. Proc. Natl. Acad. Sci. U S A 112, 14084-14089. doi: 10.1073/pnas. 1509795112

Williams, G., King, J., Cunningham, M., Stephan, M., Kerr, B., and Hersh, J. H. (2001). Fetal valproate syndrome and autism: additional evidence of an association. Dev. Med. Child Neurol. 43, 202-206. doi: $10.1017 / \mathrm{s} 001216220100038 \mathrm{x}$

Williams, P. G., and Hersh, J. H. (1997). A male with fetal valproate syndrome and autism. Dev. Med. Child Neurol. 39, 632-634. doi: 10.1111/j.1469-8749.1997. tb07500.x

Yawata, S., Yamaguchi, T., Danjo, T., Hikida, T., and Nakanishi, S. (2012). Pathway-specific control of reward learning and its flexibility via selective dopamine receptors in the nucleus accumbens. Proc. Natl. Acad. Sci. U S A 109, 12764-12769. doi: 10.1073/pnas.1210797109

Conflict of Interest: The authors declare that the research was conducted in the absence of any commercial or financial relationships that could be construed as a potential conflict of interest.

Copyright (C) 2019 Schiavi, Iezzi, Manduca, Leone, Melancia, Carbone, Petrella, Mannaioni, Masi and Trezza. This is an open-access article distributed under the terms of the Creative Commons Attribution License (CC BY). The use, distribution or reproduction in other forums is permitted, provided the original author(s) and the copyright owner(s) are credited and that the original publication in this journal is cited, in accordance with accepted academic practice. No use, distribution or reproduction is permitted which does not comply with these terms. 